\title{
UNIQUENESS INTERVALS AND TWO-POINT BOUNDARY VALUE PROBLEMS
}

\author{
Grant B. Gustafson
}

\begin{abstract}
Consider a linear $n$th order differential equation with continuous coefficients and continuous forcing term. The maximal uniqueness interval for a classical 2-point boundary value problem will be calculated by an algorithm that uses an auxiliary linear system of differential equations, called a Mikusinski system. This system always has higher order than $n$. The algorithm leads to a graphical representation of the uniqueness profile and to a new method for solving 2-point boundary value problems. The ideas are applied to construct a graphic for the conjugate function associated with the $n$th order linear homogeneous differential equation. Details are given about how to solve classical 2-point boundary value problems, using auxiliary Mikusinski systems and Green's function.
\end{abstract}

\section{Introduction}

Let $L$ be an $n$th order linear differential operator with continuous coefficients given by

$$
L y \equiv y^{(n)}(t)+\sum_{i=0}^{n-1} p_{i}(t) y^{(i)}(t) .
$$

Consider the two-point boundary value problem

$$
\begin{aligned}
L y & =f(t), \\
y(a) & =y^{\prime}(a)=\cdots=y^{(p-1)}(a)=0, \\
y(b) & =y^{\prime}(b)=\cdots=y^{(q-1)}(b)=0,
\end{aligned}
$$

where $p+q=n$ and $f(t)$ is continuous.

2000 Mathematics Subject Classification: 39 B05.

Keywords: uniqueness, boundary value problem, Mikusinski system. 


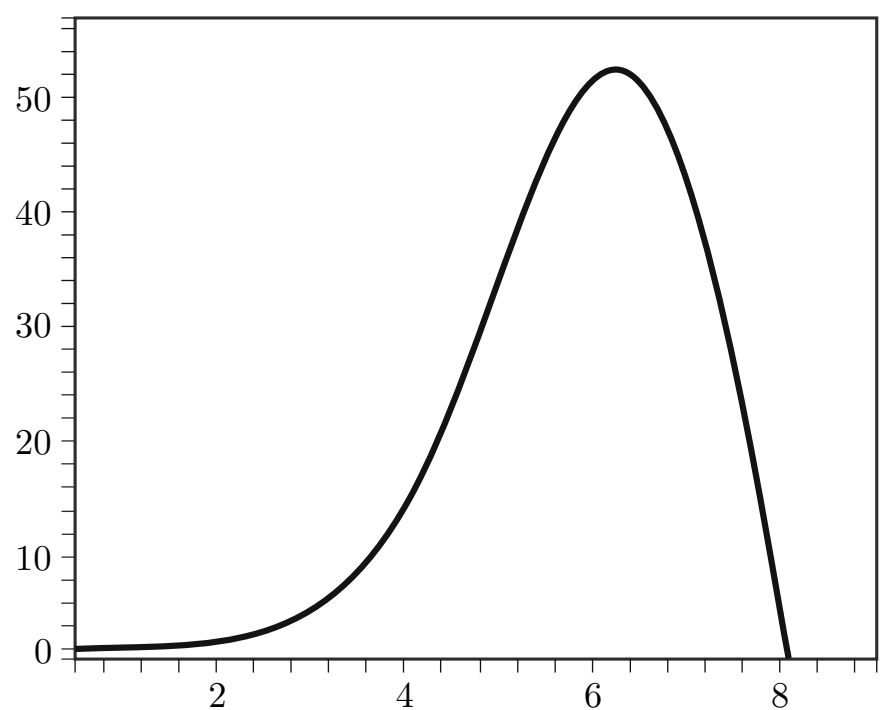

Figure 1. Uniqueness profile. Plot of $W(t)$ for the fourth order equation.

The boundary conditions are $y(0.9)=0, y^{\prime}(0.9)=0, y(b)=0, y^{\prime}(b)=0$.

The solution $y(t)$ is unique if and only if the homogeneous problem has only the solution $y(t) \equiv 0$. Let $y_{j}\left(t, t_{0}\right)$ be the unique solution of $L y=0$ with initial conditions $y_{j}^{(i)}\left(t_{0}, t_{0}\right)=\delta_{i j}$ and define $W(t)$ to be the Wronskian determinant of the functions $y_{j}(t, a)$ for $j=q, \ldots, n-1$. Then a nontrivial solution of $L y=0$ exists satisfying the boundary conditions at $t=a$ and $t=b$ if and only if $W(b)=0$. The interval $a \leq t \leq b_{1}$ is called a uniqueness interval provided $W(b) \neq 0$ for $a<b<b_{1}$, but $W\left(b_{1}\right)=0$.

A uniqueness profile is a plot of the function $W(t)$ on some subinterval of $a \leq t<\infty$. The first crossing of $W(t)$ with the $t$-axis defines the maximal interval of uniqueness of the 2-point boundary value problem. Shown in Figure 1 is a uniqueness profile for the boundary value problem

$$
\begin{gathered}
y^{i v}=p(t) y^{\prime \prime}, \\
y(a)=y^{\prime}(a)=0, \\
y(b)=y^{\prime}(b)=0 .
\end{gathered}
$$

The piecewise continuous function $p(t)$ equals -1 on $\frac{3 \pi}{2}<t<\infty$ and zero elsewhere, except for a small linear connection interval. 


\section{Mikusinski system}

The presentation will concentrate on the case of a fourth order differential equation

$$
y^{i v}=p_{0}(x) y+p_{1}(x) y^{\prime}+p_{2}(x) y^{\prime \prime}+p_{3}(x) y^{\prime \prime \prime}
$$

and the boundary conditions

$$
y(a)=y^{\prime}(a)=0, \quad y(b)=y^{\prime}(b)=0,
$$

with specializations $p_{0}=p_{1}=p_{3}=0$ and $p_{2}=p(t)$.

Let $w\left(n_{1}, n_{2}\right)$ denote the Wronskian of $y_{0}^{\left(n_{1}\right)}(t), y_{1}^{\left(n_{2}\right)}(t)$. Define state variables by the equations

$$
\begin{aligned}
& T_{1}=w(0,1), \\
& T_{2}=w(0,2), \\
& T_{3}=w(0,3), \\
& T_{4}=w(1,2), \\
& T_{5}=w(1,3), \\
& T_{6}=w(2,3) .
\end{aligned}
$$

Then determinant differentiation rules and use of the fourth order differential equation imply the Mikusinski system

$$
\begin{aligned}
& T_{1}^{\prime}=T_{2}, \\
& T_{2}^{\prime}=T_{3}+T_{4}, \\
& T_{3}^{\prime}=T_{5}+p(x) T_{2}, \\
& T_{4}^{\prime}=T_{5}, \\
& T_{5}^{\prime}=T_{6}+p(x) T_{4}, \\
& T_{6}^{\prime}=0 .
\end{aligned}
$$

Because of the known initial conditions for $y_{j}^{(i)}$, the initial data for the state variables is

$$
T_{1}(a)=0, \quad T_{2}(a)=0, \quad T_{3}(a)=0, \quad T_{4}(a)=0, \quad T_{5}(a)=0, \quad T_{6}(a)=1 .
$$

In summary, the uniqueness profile for the fourth order boundary value problem on $a \leq t<\infty$ is the plot of the unique solution of component $T_{1}$ of the sixth order Mikusinski system $T^{\prime}=M T$ with initial condition $T(a)$ equal to the last column of the identity matrix. 
Remarks. The Mikusinski system $T^{\prime}=M T$ can be defined for any set of 2-point boundary conditions. The initial data $T(a)$ is always the last column of the identity matrix. The algorithm for computer algebra generation of $M$ appears in Gustafson-Ridenhour [2].

Jan Mikusiński (1913-1987) is credited with the idea of using Wronskians to discover properties of solutions of linear differential equations, hence the naming convention for the system $T^{\prime}=M T$. It is not claimed that this system is first identified here, because in truth we ( $\mathrm{Rid}$ en h o u r - G u s t a f s o n ) discovered it during computations done in 1988.

What is claimed for state variable $T_{1}$ is also true for all other state variables. In the case of constant coefficients $p_{j}$, each state variable is a solution of a linear constant-coefficient differential equation $\mathbf{M}_{0} y=0$, where the characteristic equation of $\mathbf{M}_{0}$ equals the characteristic equation $\operatorname{det}(M-\lambda I)=0$ of the Mikusinski system $T^{\prime}=M T$. To prove the claim, multiply the Cayley-Hamilton matrix identity by $T$ and use $T^{(r)}=M T^{(r-1)}, 1 \leq r \leq n$. Equation $\mathbf{M}_{0} y=0$ can be selected to have even lower order, by using the minimal polynomial matrix identity, instead of the characteristic polynomial matrix identity.

\section{Conjugate functions and uniqueness}

Following P. Hartman [3, 4] and T. Sherman [7], the first conjugate point of $L y=0$ to $t=a$ is the least number $b>a$ such that on $a \leq t \leq b$ there is a nontrivial solution with $n$ zeros counting multiplicities. It is known that either $b=\infty$ or else $b=\eta_{1}(a)$ is a continuous function of the point $a$.

A more precise description of $\eta_{1}$ is possible, in terms of the nontrivial solutions of 2-point boundary value problems. Define $b(a, k)$ to be the first crossing greater than $a$ of the uniqueness profile for boundary conditions $y(a)=\cdots$ $\cdots=y^{(k-1)}(a)=0, y(b)=\cdots=y^{(n-k-1)}(b)=0$, for $k=1, \ldots, n-1$. If there is no first crossing, then define $b(a, k)=\infty$. Then (see Sherman [6])

$$
\eta_{1}(a)=\min \{b(a, k): 0 \leq k \leq n-1\} .
$$

The minimization implies some continuity properties of $\eta_{1}$, but also there is generally a corner in the graph of $\eta_{1}$, a point of non-differentiability, each time the minimum switches from $b(a, i)$ to $b(a, j)$ with $i \neq j$. This typical behavior is illustrated in Figure 2, which is a plot of $\eta_{1}(a)$ from $a=0$ to $a=9$ for the sixth order equation

$$
y^{(6)}=p(t) y^{(4)}
$$




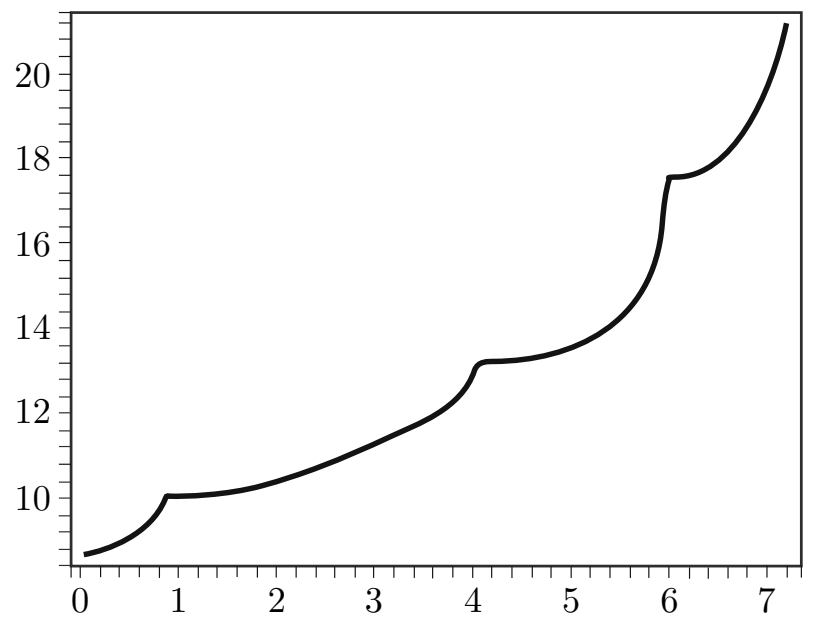

Figure 2. A typical conjugate function plot. The sixth order equation is used to illustrate the piecewise continuous character of $\eta_{1}$. The piecewise sections are realized by $(4,2),(3,3),(2,4)$ and $(5,1)$ boundary value problems, respectively.

For illustration purposes, coefficient $p(t)$ is piecewise linear, with $p(t)=-1$ on $\frac{3 \pi}{2}<t<3 \pi$ and zero elsewhere, except on two small linear connection intervals.

Remark. The Figure 2 shows that the conjugate function for a constant-coefficient differential equation is not the typical case. For such equations, $\eta_{1}(0)=$ $b(0, k)$ for some $k$. Translation-invariance of constant equations then implies that $\eta_{1}(a)=b(a, k)$. Accordingly, there are no corners in the graph of $\eta_{1}$. Furthermore, continuity of $\eta_{1}$ then implies continuity of $b(\cdot, k)$, which is not true for non-constant coefficients.

\section{Green's function and uniqueness profiles}

The Green's function $G(t, s)$ for the boundary value problem

$$
\begin{gathered}
L y=f(t), \\
y(a)=y^{\prime}(a)=\cdots=y^{(p-1)}(a)=0, \\
y(b)=y^{\prime}(b)=\cdots=y^{(q-1)}(b)=0,
\end{gathered}
$$


where $p+q=n$, can be defined as follows. Let $u_{0}(t, s), \ldots, u_{n-1}(t, s)$ be the fundamental set of solutions to $L y=0$ defined by $u_{i}^{(j)}(s, s)=\delta_{i j}$ (standard basis). Then

$$
G(t, s)=\frac{\operatorname{det} B(t, s)}{\operatorname{det} W},
$$

where the matrices $B$ and $W$ are given by:

$$
\begin{gathered}
W=\left[\begin{array}{ccc}
u_{q}(b, a) & \cdots & u_{n-1}(b, a) \\
u_{q}^{\prime}(b, a) & \cdots & u_{n-1}^{\prime}(b, a) \\
\vdots & & \vdots \\
u_{q}^{(p-1)}(b, a) & \cdots & u_{n-1}^{(p-1)}(b, a)
\end{array}\right], \\
B(t, s)=\left[\begin{array}{cccc}
K(t, s) & u_{q}(t, a) & \cdots & u_{n-1}(t, a) \\
u_{n-1}^{\prime}(b, s) & u_{q}^{\prime}(b, a) & \cdots & u_{n-1}^{\prime}(b, a) \\
\vdots & \vdots & & \vdots \\
u_{n-1}^{(p-1)}(b, s) & u_{q}^{(p-1)}(b, a) & \cdots & u_{n-1}^{(p-1)}(b, a)
\end{array}\right],
\end{gathered}
$$

where $K(t, s)=0$ for $t<s$, and $K(t, s)=u_{n-1}(t, s)$ for $t \geq s$. Verified in Gustafson-Ridenhour [2] is the formula

$$
G(t, s)=K(t, s)+H(t, s),
$$

where $H(t, s)$ is defined by

$$
H(t, s)=\sum_{i=0}^{p-1} v_{i}(s) u_{q+i}(t, a)
$$

and

$$
v_{i}(s)=\sum_{j=0}^{q-1}(-1)^{1+i} z_{i j} c_{j}(s)-c_{q+i}(s) .
$$

The symbols $z_{i j}$ are constants, initially defined as quotients of determinants, but ultimately computed as quotients of components of the Mikusinski system evaluated at $t=b$. The functions $c_{j}$ are a standard solution basis for the formal adjoint system.

Function $v_{i}$ can be computed from its initial values by a numeric initial value problem solver, such as RK4 or LSODE. The initial values of $v_{i}$ are expressed entirely in terms of the constants $z_{i j}$, which are values of components of the Mikusinski system.

The end result is that $G(t, s)$ can be computed using initial value solvers applied to the system and its adjoint system, provided some one-time initial value solver computations $z_{i j}$ are done. 


\section{UNIQUENESS INTERVALS}

The solution of the boundary value problem for $L y=f$ can be expressed as

$$
y(t)=\int_{a}^{b} G(t, s) f(s) \mathrm{d} s .
$$

Because $G(t, s)$ can be computed for any pair $(t, s)$, then quadrature rules can be applied to reduce the computational effort to a small number of points in $a \leq t \leq b$, for example, Gaussian quadrature 40-point rules.

\section{REFERENCES}

[1] GUSTAFSON, G. B.-RIDENHOUR, J. R.: Lower order branching and conjugate function discontinuity, J. Differential Equations 27 (1978), 167-179.

[2] GUSTAFSON, G. B.-RIDENHOUR, J. R.: Uniqueness intervals for multipoint boundary value problems (preprint 2008).

[3] HARTMAN, P.: Unrestricted n-parameter families, Rend. Circ. Mat. Palermo (2) 7 (1958), 123-142.

[4] HARTMAN, P.: Ordinary Differential Equations, John Wiley and Sons, Inc., New York, 1964.

[5] MIKUSIŃSKI, J.: Sur l'équation $x^{(n)}+A(t) x=0$, Ann. Polon. Math. 1 (1955), 207-221.

[6] SHERMAN, T. L.: Properties of solutions of Nth order linear differential equations, Pacific J. Math. 15 (1965), 1045-1060.

[7] SHERMAN, T. L.: Conjugate points and simple zeros for ordinary linear differential equations, Trans. Amer. Math. Soc. 146 (1969), 397-411. 